\title{
Exploratory-Data and Statistical Analyses of AB070597, an Amino Acid/ Peptide Complex, on Blood-Serum Creatinine Concentration and Estimated Glomerular Filtration Rate: A Non-Randomized Pilot Trial of Five Humans with Declining Renal Function
}

\section{James D. Archer* (D) \\ ${ }^{1}$ Applied Research Laboratory, \\ Villa Pompeii Campus 4848 Olive Hill Road (28 Rolling View) Fallbrook, CA 92028, USA}

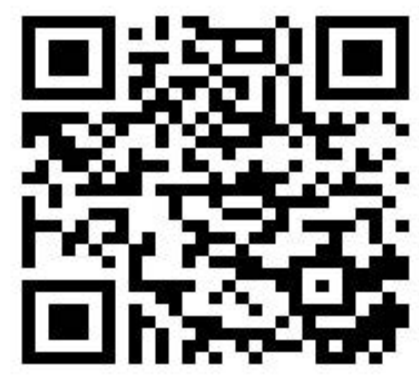

Keywords:

Glomerular, Kidney Disease, Amino Acid, Progression

\section{Abstract}

Background:

Chronic kidney disease (CKD) develops from persistent, irremediable loss of renal function (RF). Animal studies show that dietary supplement, AB070597, an amino acid/ peptide complex, can stabilize, and in some instances, reverse CKD. Previous human CKD studies demonstrate that dietary treatment with specific keto acids and amino acids can lower blood-serum creatinine concentration (SCr) and increase glomerular filtration rate (GFR). This pilot trial was performed to determine whether a future randomized controlled human clinical trial of AB070597's effect on CKD was justified. Methods:

The trial was structured as a consecutive case series to evaluate whether oral treatment with $\mathrm{AB} 070597$ could slow $\mathrm{CKD}$ progression, as gaged by $\mathrm{SCr}$, and estimated glomerular filtration rate (eGFR). Eligibility requirements: Nondiabetic white males under current medical care in the United States, diagnosed with $\mathrm{CKD}$, or with histories of increasing $\mathrm{SCr}$ and declining eGFR, resulting from CKD or natural consequence of aging. Exclusion criteria: Concurrent or suspected comorbidities unrelated to CKD. Participants ingested 6-g bi-daily doses of $\mathrm{AB} 070597$ for periods up to 24 months. SCr was measured and eGFR calculated at approximate tri-monthly intervals.

Results:

Results were presented as individual-participant and group SCr and eGFR vectors, and initial-data-analysis diagrams.Treatment reversed $\mathrm{SCr}$ slopes from positive to negative, and eGFR slopes from negative to positive. When individual participant's median monthly eGFR rate-of-change (positive) was compared with the median monthly eGFR rate-of-change (negative) in a sample of 2870 humans with CKD, differences were significant in favor of the $\mathrm{AB} 070597$ treatment group in 4 of 5 participants: $(\mathrm{P}=0.0625,95 \%$ CI:0.368-8.368), ( $\mathrm{P}=0.0313,95 \%$ CI: 0.368-1.038), ( $\mathrm{P}=0.0039$, 95\% CI: 0.368-0.698),

( $\mathrm{P}=0.0010,95 \%$ CI:0.118-2.868), ( $\mathrm{P}<0.0001,95 \%$ CI: 0.368-10.370).

Conclusion:

Oral AB070597 treatment produced an apparently favorable change in CKD trajectory in 4 humans and in 1 with naturally age-related declining RF. The magnitude and direction of change hint that treatment may have had a beneficial effect on CKD progression and age-related RF. In and of themselves, this pilot trial's results seem to favor CKD treatment with AB070597, but because there was no randomization, no control group, and a small sample size, it is not possible to extend results beyond its bounds. They do, however, support the rationale for a future randomized controlled trial.

Key points:

-AB070597 may be a useful clinical tool for the management of CKD and naturally declining RF in humans.

- No serious adverse effects were reported. 


\section{1 | INTRODUCTION}

$\mathrm{T}$ his trial explored the influence of bi-daily oral doses of AB070597 on SCr and eGFR in a small group of individuals with histories of rising $\mathrm{SCr}$ and negatively correlated eGFR.

CKD develops from persistent, irremediable loss of RF. Complicating dynamics, such as hypertension, anemia, renal-osteodystrophy, pericarditis, cardiovascular disease are common sequelae; followed by end-stage renal failure, and death. Currently, 37 million American adults have CKD, and 1 in 3 is at risk due to diabetes mellitus, hypertension, heart disease, obesity, or genetics (1). United States Centers for Disease Control and Prevention data reveal that CKD prevalence is $38 \%$ in individuals over 65 years old, $13 \%$ in those $45-64$ years of age, $7 \%$ in those 18 44 years old, and is $15 \%$ in women versus $12 \%$ in men. $16 \%$ of non-Hispanic blacks, $13 \%$ of nonHispanic whites, and 14\% of Hispanics have CKD [2]. Diabetes mellitus and hypertension are the main causative factors $(2,3)$.

Progressively declining GFR is the hallmark of CKD. GFR declines predictably in most humans with advancing age (4) . Acute physical trauma to renal tissue, infection, and drug or chemical-induced nephrotoxicity can cause GFR to fall precipitously, but values usually return to normal, or near-normal, with proper medical intervention. On the other hand, GFR does not normalize in patients who develop CKD. GFR continues to decay, and irrespective of the primary cause, evolves to progressive tubulointerstitial fibrosis with deteriorating RF and reduced nephron count; thus, its measurement becomes a useful means for judging RF and CKD status. Direct GFR measurement is the benchmark for assessing renal performance; but direct measurement requires multiple urine and blood-sample collections; along with continuous intravenous infusion of inulin, and hence does not easily lend itself to clinical implementation. In order to surmount this limitation, an alternate indirect method was developed to estimate GFR by utilizing steady-state $\mathrm{SCr}$, a metabolite of creatine phosphate, which is found mainly in muscle; along with other factors such as age, race, and gender. Indirectly measured GFR is referred to as estimated glomerular filtration rate (eGFR). $\mathrm{SCr}$ and calculated eGFR were the parameters measured in this investigation.

Blood-serum amino acid concentrations in humans with CKD are abnormal, when compared to concentrations in those with normal RF. Abnormal values include increased L-citrulline and decreased L-arginine, which correlate with elevated $\mathrm{SCr}$ (5, 6). Renal L-arginine synthesis is also markedly reduced (7). Plasma L-glutamine and L-histidine are notably lower $(8,9)$. Plasma asparagine levels decline progressively in patients with inflammation, malnutrition, or both (10); and finally, intracellular L-carnosine is reduced in uremic patients (11). Previous studies show that oral treatment with certain keto acids and amino acids arrest time-related $\mathrm{SCr}$ elevations in some CKD patients $(12,13)$. Dietary L-arginine supplementation increases GFR (14) L-carnosine augments bone morphogenetic protein7 (BMP-7) gene expression in human periodontal ligament cells (15). Zeisberg et al., demonstrated that BMP-7 counteracts transforming growth factor-B1-induced epithelial-to-mesenchymal transition, thereby reversing chronic kidney injury (16) In a study by Archer (2019), human primary proximal renal tubule epithelial cells (hPRTEC) incubated with L-carnosine, at $1.763 \mathrm{ug} / \mathrm{ml}$, increased BMP-7 messenger RNA (mRNA) expression 2.13-fold (17). L-carnosine is not detectable in blood-serum by current assay methods in fasted humans $(18,19)$.

Various amino acid concentrations are also abnormal in cats with CKD (20). In a previous study, cats with CKD experienced a significant median $\mathrm{SCr}$ reduction over 104 weeks $(\mathrm{P}=0.0084)$, as well as a significant reduction in mean International Renal Interest Society (IRIS) disease stage $(\mathrm{P}=0.0184)$ when treated orally with AB070597. Moreover, hPRTECs incubated with AB070597 at $3.125 \mathrm{ug} / \mathrm{ml}$,

Supplementary information The online version of this article (https://doi.org/10.15520/jcmro.v3i11.36 7) contains supplementary material, which is available to authorized users.

Corresponding Author: James D. Archer

Applied Research Laboratory, Villa Pompeii Campus 4848 Olive Hill Road (28 Rolling View) Fallbrook, CA 92028, USA

Email:jarcher@appliedresearchlaboratory.us 
showed a 3.29-fold increase in BMP-7 mRNA expression (17).

Based on the foregoing information, we hypothesized that oral treatment with AB070597 might have potential to stabilize or lower $\mathrm{SCr}$, and recover RF in humans with CKD, or in those with naturally declining RF. AB070597 was formulated as a compound of amino acids and a peptide, chosen to address the deficits discussed above, and to increase exogenous BMP-7 expression. The amino acids and peptide in AB070597 are commercially available and are generally regarded as safe when taken individually by humans as dietary supplements. Previous studies demonstrated the safety of these combined ingredients in cats (20) and in dogs (21). A study by Archer, J. (2019) found no AB070597 cytotoxicity in cell viability assays using hPRTEC (17).

\section{2 | 2 MATERIALS AND METHODS}

\section{1 | Design}

This trial was structured as a consecutive case series to determine if oral dosing with AB070597 could slow CKD progression, as gaged by $\mathrm{SCr}$ and calculated eGFR.

\section{2 | Participants}

Participants were recruited from Southern California medical practices from November 2016 to November 2017. Data collection occurred from November 2016 to November 2018. Ages ranged from 63 to 80 years. Participants were identified as [AB070597n $(\mathrm{xx})]$, where $\mathrm{n}=0-4$, and where $\mathrm{xx}=$ age in years at trial entry: AB07059700 (74), AB07059701 (63), AB07059702 (80), AB07059703 (66), and AB07059704 (66). Eligibility requirements: Nondiabetic white males under current medical care in the United States, diagnosed with CKD or with a history of increasing $\mathrm{SCr}$ resulting from CKD or natural consequence of aging. Exclusion criteria: Concurrent or suspected comorbidities unrelated to CKD. Two participants, AB07059700 and AB07059701, were prescribed low-protein diets by their supervising nephrologists. Participants were instructed to dissolve the contents of 6 capsules of AB070597 in a one-half cup of water or fruit/ vegetable juice and consume as a beverage, once in the morning and once in the evening, for a total of $12 \mathrm{~g} /$ day. Physician-prescribed medications were allowed, and non-prescription supplements or herbal remedies were prohibited. Pre-trial retrospective $\mathrm{SCr}$ and eGFR data, and time-in-trial varied among participants.

\section{3 | AB070597}

AB070597 amino acids and peptide weights were extrapolated from human and animal studies that showed positive correlation with improved RF in CKD subjects. Amino acids and peptide were supplied, compounded as capsules, bottled, and labeled by Albert Max, Inc., a United States Food and Drug Administration registered manufacturing facility (Chino, California, USA). Each 1-g capsule included $83 \mathrm{mg}$ L-arginine, $167 \mathrm{mg}$ glycine, 167 mg L-glutamine, $83 \mathrm{mg}$ L-histidine, $167 \mathrm{mg} \mathrm{L}$ aspartic acid, $167 \mathrm{mg}$ L-glutamic acid, and $167 \mathrm{mg}$ L-carnosine.

\section{4 | Body Weight}

A digital automatic-calibration body-weight scale (EatSmart Products, \#: ESBS-05, Oak Brook, Illinois, USA) was issued to each participant, along with a daily diary to document dosing compliance, record weekly body-weight measurements, and any unusual symptoms.

\section{5 | Blood-Sample Collection and Laboratory Measurements}

SCr was measured and eGFR calculated approximately every 3 months, up to 24 months or participant trial departure. Blood samples $(5 \mathrm{~mL})$ were collected at local Quest Diagnostics, Inc. clinics (Corporate Headquarters, 500 Plaza Dr., Secaucus, NJ 07094). Measurements from the first clinic visit (pre-dosing) served as baselines for $\mathrm{SCr}$ and eGFR for each participant. eGFR was assessed by $\mathrm{SCr}$, utilizing the Chronic Kidney Disease Epidemiology Collaboration formula (22). Participants' diaries 


\section{CURRENT MEDICAL RESEARCH AND OPINION}

were collected at trial end or departure.

\section{6 | Safety}

Safety was assessed using adverse event (AE) reporting. An AE was defined as an unexpected medical event occurring during treatment, or untoward medical occurrence, which did not necessarily have a causal relationship with treatment. Expected AEs included early-on transient gastrointestinal distress. AEs were collected from the time a participant took his first dose until the final clinic visit. AEs were identified through observation, participant comment or question. AEs were followed until they resolved, were clinically stable, or until 30 days after a participant departed the trial.

\section{3 | STASTICAL ANALYSIS}

Pre-treatment and treatment vectors were plotted to visualize any $\mathrm{SCr}$ or eGFR slope change. Vectors were also plotted to picture changes from treatmentstart baselines to study end (exploratory data analysis [IDA]).

A non-parametric one-sample Wilcoxon signed-rank test (initial data analysis [IDA]) was used to compare participant eGFR median-rate-of-change per unit time to eGFR median-rate-of-change per unit time of 2870 CKD patients in a study by Tsai et al. (23), at 0.05 significance, using GraphPad Prism (San Diego, California, USA). Post-trial statistical power was calculated from Tsai et al. (24) eGFR rate-of-change mean; trial treatment eGFR rate-ofchange mean, and pooled standard deviation at 0.05 significance (DSS Research, https://www.dssresearc h.com). The probability of an individual participant attaining a particular study-end vector state or trend was calculated by comparing their study-end vector state to all possible vector states or trends

\section{4 | 4 RESULTS}

\section{1 | 4.1 Statistical Analyses: Exploratory Data Analysis, Initial Data Analysis}

At treatment baseline (vector head-tail intersections) Figure 1Figure 2, SCr vector slopes changed from positive to negative, and eGFR vector slopes changed from negative to positive for all participants. Figure 3 shows that $\mathrm{SCr}$ decreased from treatment baseline versus time for all participants. Figure 4 illustrates the opposite trend for eGFR, with rates increasing from treatment baseline for each participant. The one-sample Wilcoxon signed-rank test quantified significant eGFR decline rate reversals in 4 of 5 participants (Figure 5 , Table 1 ).

TABLE 1: WilcoxonSigned-Rank Test of eGFR Decline Rate Reversal (Overall Post-Trial Statistical Power $=99.2 \%$ )

\begin{tabular}{lll}
$\begin{array}{l}\text { Participant } \\
\text { ID }\end{array}$ & $\begin{array}{l}95 \% \text { Confidence } \\
\text { Intervals }\end{array}$ & P-value \\
\hline$A B 07059700$ & $0.368-8.368$ & 0.0625 \\
AB07059701 & $0.368-1.038$ & $0.0313^{*}$ \\
AB07059702 & $0.368-0.698$ & $0.0039^{*}$ \\
AB07059703 & $0.118-2.868$ & $0.0010^{*}$ \\
AB07059704 & $0.368-10.370$ & $<$ \\
& & $0.0001^{*}$ \\
\hline
\end{tabular}

* = significant at $\mathrm{P} \leq 0.05$

The compound probability of arriving at the group's study-end vector-state gave insight as to whether the observed treatment effect was true (i.e., plausible by $\mathrm{P}$ value). There were 5 possible SCr and eGFR studyend vector-states and trends: 1) no change from pre-treatment slope (i.e., no treatment effect), 2) increasing slope, 3) decreasing slope, 4), steady-state (vector slope $=0$, progression halted), and 5) pretreatment and treatment slope reversals (i.e., CKD reversal) . The probability of a single participant's study-end vector-state or trend was $1 / 5$. The compound probability of all 5 participants attaining $\mathrm{SCr}$ and eGFR slope reversals was $(1 / 5)^{\mathbf{5}}=0.00032$ (i.e., $\mathrm{P}<0.001)$. 


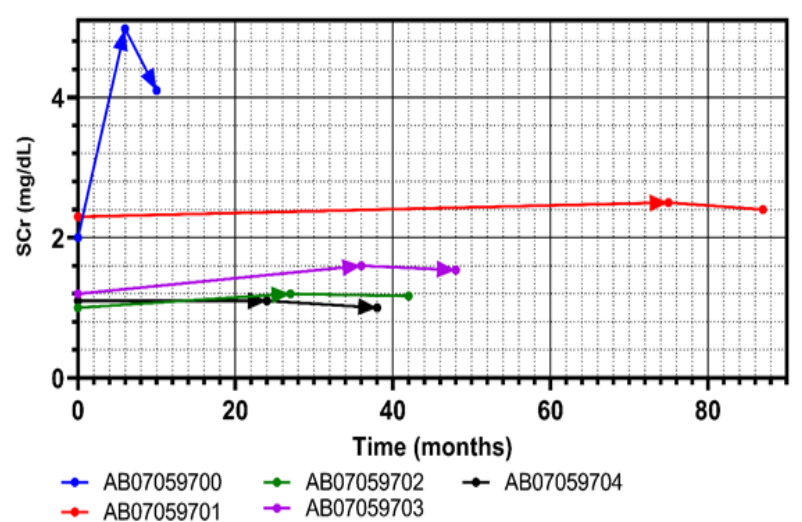

FIGURE 1: $\mathrm{SCr}^{a}$ Pre-Treatment and Treatment Vectors

$\mathrm{a}=$ blood-serum creatinine concentration. Pretreatment $\mathrm{SCr}$ vector slopes were sign-positive from trial baseline $(x$-axis $=0$ ) to treatment baseline (vector head-tail intersections) for allparticipants, then vector slopes reversed to sign-negative.

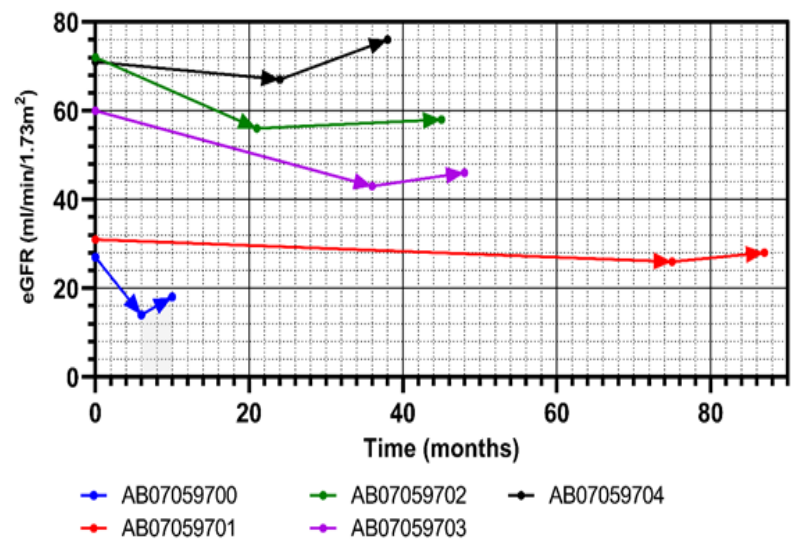

FIGURE 2: $\mathrm{eGFR}^{a}$ Pre-Treatment and Treatment Vectors

$\mathrm{a}=$ estimated glomerular filtration rate. Pre-treatment eGFR vector slopes weresign-negative from trial baseline( $x$-axis $=0$ ) to treatment baseline (vector head-tail intersections) for allparticipants, then vector slopes reversed to sign-positive.

\section{2 | Safety}

Overall, AB070597 was well tolerated by participants. AB07059703 and AB07059704 experienced mild diarrhea for 1 and 2 days, respectively. No other AEs were reported.

\section{3 | Body Weight}

Participant body weights did not change notably during the treatment period (data not shown).

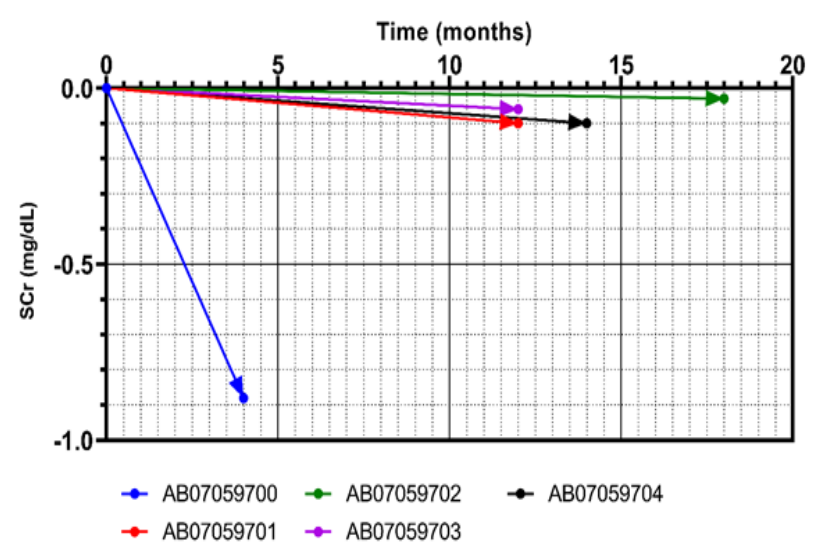

FIGURE 3: $\mathrm{SCr}^{a}$ : Change from Treatment-Baseline Vectors

$\mathrm{a}=$ blood-serum creatinine concentration. $\mathrm{SCr}$ declined from treatment baseline $(x$-axis $=0)$ forall participants.

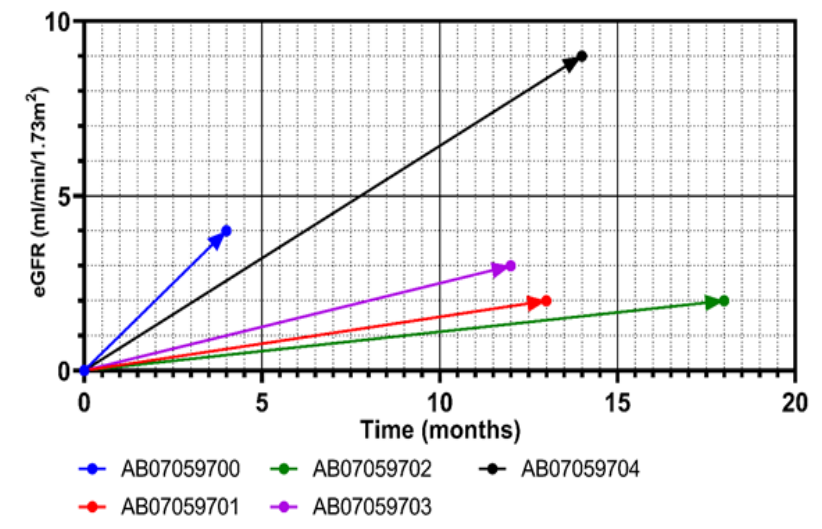

FIGURE 4: eGFR ${ }^{a}$ Change from Treatment-Baseline Vectors

$\mathrm{a}=$ estimated glomerular filtration rate. eGFR increased from treatment baseline for allparticipants.

\section{5 | DISCUSSION}

Examination of disease progression in individuals can reveal information and offer insights that the frequentist approach to data analysis (e.g., models and hypothesis tests of groups) sometimes obscures. Tukey (1977) developed methods for visually observing data to grasp overall structure (25). This trial used EDA, as set forth by Tukey, in the form of $\mathrm{SCr}$ and eGFR vectors, in conjunction with the IDA non-parametric one-sample Wilcoxon signedrank test, to analyze the effect of oral AB070597 supplementation on SCr and eGFR in 5 humans with declining RF.

EDA is a statistical approach to gain visual insight to the shape and structure of data-sets beyond that 


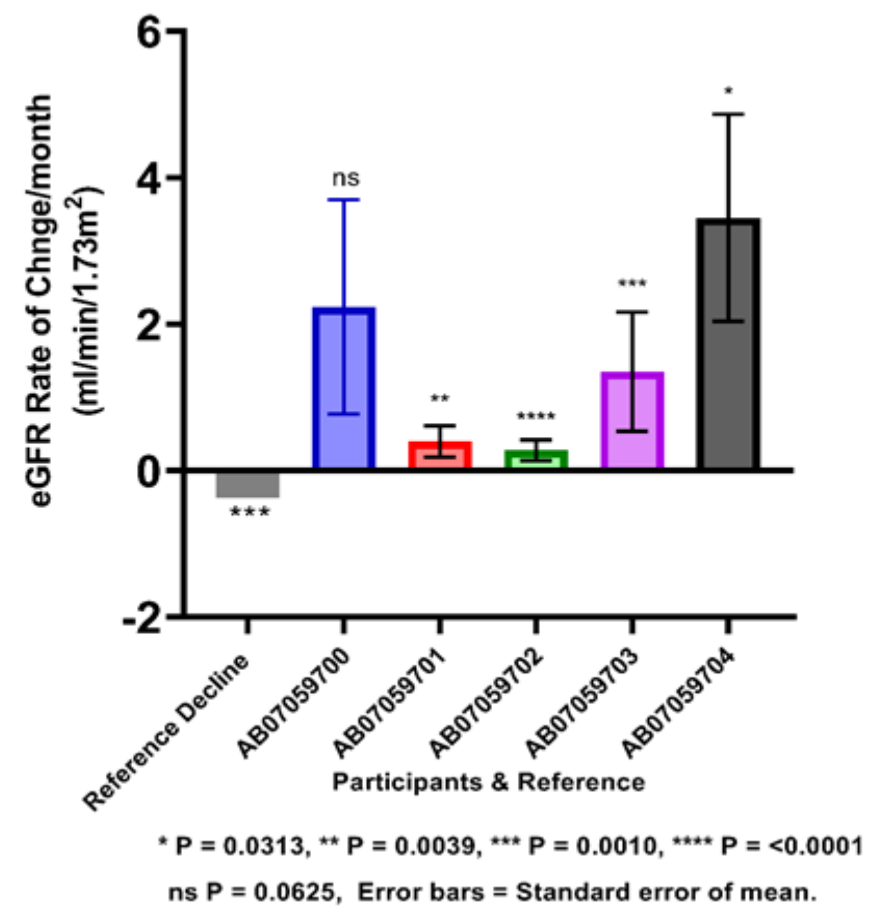

FIGURE 5: Median eGFR ${ }^{a}$ Treatment Rate of Change per Month

$\mathrm{a}=$ estimated glomerular filtration rate. Grey bar represents median monthly eGFR decline in 2870 humans with CKD in a study by Tsai et al. (24) .Blue, red, green, purple, and black bars represent median monthly eGFR increasesfor this trial's participants.

which formal modeling or hypothesis testing can reveal. EDA was chosen for its ability to visually display pre-treatment and treatment-interval $\mathrm{SCr}$ and eGFR vector-slopes and trends. IDA was selected because it made conventional hypothesis test calculations possible (i.e., $\mathrm{P}$ values), thus, offering a way to correlate visual observations with a nullhypothesis.Figure 1 and Figure 2 graphically display each participant's $\mathrm{SCr}$ and eGFR vector-state during 2 important intervals: 1) pre-treatment baseline $(\mathrm{x}$-axis $=$ time 0$)$ to treatment baseline (headtail intersection), and 2) treatment baseline to final laboratory measurement (head-tail intersection to head). Visual comparisons of $\mathrm{SCr}$ and eGFR vector slopes revealed uniform treatment responses by all participants, with $\mathrm{SCr}$ moving from positive (declining RF), to negative (improving RF). eGFR vectors moved from negative to positive, as would be expected, since eGFR correlates negatively with
SCr. Each participant's SCr and eGFR vectors represented the mean of all vectors that followed CKD progression between laboratory measurements, and as such, did not give precise information about how $\mathrm{SCr}$ and eGFR moved along the pathway from pretreatment baseline to final laboratory measurement. With all data available, it would have been possible to display all incremental vectors, but in the spirit of EDA, we chose to display mean vectors to gain the overall view. SCr and eGFR change-from-treatment baselines vectors Figures 3 and 4 presented the uni-form response of participants to treatment, with SCr decreasing and eGFR increasing, versus time.

Mean vectors, as opposed to vectors constructed as linear regression lines, were used for analysis in order to give the most accurate assessment of treatment effect. Regression line vectors would have moved vector heads and tails from their laboratory measured values, thereby changing pre-treatment and treatment baselines, as well as the final measurement points. All participants shared similar SCr and eGFR vector slope signs at the final clinic visit; with the combined probability of such an occurrence at $\mathrm{P}<$ 0.001. Parallel analyses by EDA and IDA gave a visually compelling picture of treatment effect on CKD progression and its effect on age-related RF, as well as a graphical display with associated P-values.

These observations contrast sharply with common $\mathrm{SCr}$ and eGFR trajectories in patients with CKD or naturally declining RF, wherein SCr and eGFR increase and decrease, respectively. Biological mechanisms that relate to AB070597's composition exist that might explain these contrasts. For instance, $\mathrm{SCr}$ positively correlates with blood-serum concentrations of endogenous nitric oxide synthase inhibitors, asymmetrical dimethylarginine (ADMA) and symmetrical dimethylarginine (SDMA), in humans with CKD (26). ADMA inhibits nitric oxide synthase directly, while SDMA limits nitric oxide synthesis by acting as a competitive inhibitor of L-arginine transport (27). L-arginine in AB070597 may have contributed to increased nitric oxide production since it is its unique biological substrate. Zoccali et al. described the pathogenic role of blocked nitric oxide synthesis in humans and its negative impact on RF (28). L-arginine supplementation consistently improves GFR in diverse models of renal disease 
in humans (29). Glycine infusion increases effective renal plasma flow and GFR in rats and reduces proximal and distal tubule $\mathrm{Na}+$ reabsorption (30). The deleterious effects of reactive oxygen species (ROS) production in renal cells is generally accepted. L-aspartic and L-glutamic acids are efficient ROS scavengers (31). Glutamine suppresses proteolysis and stimulates protein synthesis in skeletal muscle (32). L-histidine is an essential amino acid in normal and chronically uremic humans (33). Lastly, L-carnosine-increased BMP-7 gene expression might have improved RF by inducing nonfunctional nephrons to differentiate and regain their normal phenotype, thus increasing the functional nephron number.

\section{6 | CONCLUSIONS}

AB070597 treatment produced an apparently favorable change in CKD trajectory, as gauged by $\mathrm{SCr}$ and eGFR in 4 humans with CKD and in 1 with naturally declining RF. The magnitude and direction of change hints that treatment may have had a beneficial effect on the progression of CKD and age-related RF. In and of themselves, this pilot trial's results seem positive, but because there was no randomization, no control group, and a small sample size, it is not possible to extend results beyond its bounds. They do, however, support further investigation of AB070597, and forms the rationale for a randomized controlled trial with greater treatment and control groups.

7 ACKNOWLEDGEMENTS: The author gratefully acknowledges the staff at Whitsell Innovations, Inc. for their assistance with the trial plan.

8 FUNDING: Major funding was provided by the John T. Fulton Trust with additional minor funding from Applied Research Laboratory.

\section{COMPLIANCE WITH ETHICAL STANDARDS}

10 CONFLICT OF INTEREST: I, the author, declare I have no competing or conflicting interests. I was, however, granted United States patents US 966,910 B2, US 10,668,037 B2 and European Patent EP 2928461 for my work on AB070597. I have assigned all rights to Bio Health Solutions, (Reno,
Nevada, USA). I have not received and will not receive payment or compensation of any kind for those assignments. I have no financial interest in Bio Health Solutions, and it did not fund or support my research, either directly or indirectly.

Open Access was sponsored by Applied Research Laboratory, Fallbrook, California, USA.

11 ETHICAL APPROVAL: In accordance with the Declaration of Helsinki and Department of Health and Human Services regulations (USA), the 'AB070597 Case Report' trial was reviewed by Pearl Independent Review Board and granted exemption (Pearl IRB 16-BOS-101) from requiring ethics approval and oversight under 45 CFR 46.102(d) and 45 CFR 46.101(b)(4). All participants signed informed consent.

12 DATA AVAILABILITY AND TRIAL REGISTRATION: The datasets generated and analyzed during this study are available from the corresponding author on reasonable request. The International Standard Randomized Controlled Trial Number assigned to this study is ISRCTN44802329.

\section{REFERENCES}

[1]. Kidney Disease: The Basics (2019) National Kidney Foundataion https:// www.kidney.org/news/newsroom/factsheets/ Kidney Disease Basics. Accessed 16 Jul 2019.

[2]. Chronic Kidney Disease in the United States (2019) Centers for Disease Control and Prevention 2019. http://www.cdc.gov/ kidneydisease/publications-resources/2019national-facts.html. Accessed 16 Jul 2019

[3]. Coresh J, Selvin E, Stevens LA, Manzi J, Kusek JW, Eggers $P$, et al. Prevalence of chronic kidney disease in the United States. JAMA 2007;298(17):2038-47.

[4]. Cohen E, Nardi Y, Krause I, Goldberg E, Milo G, Garty $\mathrm{M}$, et al. A longitudinal assessment of the natural rate of decline in renal function with age. J Nephrol 2014;27(6):635-41. 


\section{CURRENT MEDICAL RESEARCH AND OPINION}

[5]. Ceballos I, Chauveau P, Guerin V, Bardet J, Parvy P, Kamoun P, et al. Early alterations of plasma free amino acids in chronic renal failure. Clin Chim Acta 1990;188(2):101-8.

[6]. Kumar VS, Kumar BS, Kishore Babu S, Kumar TS, Sathya Sai RN, Soloman A, Kumar KA. Serum amino acid profile in chronic renal failure. Indian Journal of Nephrology 1998;Apr-Jun 8(2):52-4.

[7.] Tizianello A, De Ferrari G, Garibotto G, Gurreri G, Robaudo C. Renal metabolism of amino acids and ammonia in subjects with normal renal function and in patients with chronic renal insufficiency. J Clin Invest 1980; 65(5):1162-73.

[8.] Cobo M, Martin Gomez MA, Frutos MA, Benavides M. Glutamin concentrations in patients treated with cisplatin have a predicting value of renal failure development. Nefrologia 2007; 27(1):23-9.

[9.] Watanabe M, Suliman ME, Qureshi AR, Garcia-Lopez E, Barany P, Heimburger O, et al. Consequences of low plasma histindine in chronic kidney disease patients: associations with inflammation, oxidative stress, and mortality. Am J Clin Nutr 2008; 87:1860-6.

[10.] Suliman ME, Qureshi AR, Stenvinkel P, Pecoits-Filho R, Barany P, Heimburger O, et al. Inflammation contributes to low plasma amino acid concentrations in patients with chronic kidney disease. Am J Clin Nutr 2005; 82(2):342-9.

[11.] Bergstrom J, Furst P, Noree LO, Vinnars E. Intracellular free amino acids in muscle tissue of patients with chronic uraemia: effect of peritoneal dialysis and infusion of essential amino acids. Clin Sci Mol Med 1978; 54(1):51-60.

[12.] Mitch WE, Walser M, Steinman TI, Hill $\mathrm{S}$, Zeger S, Tungsanga K. The effect of a keto acid-amino acid supplement to a restricted diet on the progression of chronic renal failure. $\mathrm{N}$ Engl J Med 1984; 311(10):623-9.
[13.] Yatzidis H. Oral supplement of six selective amino acids arrest progression renal failure in uremic patients. Int Urol Nephrol 2004; 36(4):591-8.

[14.] Smoyer WE, Brouhard BH, Rassin DK, LaGrone L. Enhanced GFR response to oral versus intravenous arginine administration in normal adults. J Lab Clin Med 1991; 118(2):166-75.

[15.] Ito-Kato E, Suzuki N, Maeno M, Takada $\mathrm{T}$, Tanabe N, Takayama $\mathrm{T}$, et al. Effect of carnosine on runt-related transcription factor-2/ core binding factor alpha-1 and Sox9 expressions of human periodontal ligament cells. J Periodontal Res 2004; 39(3):199-204.

[16.] Zeisberg $M$, Hanai J, Sugimoto $H$, Mammoto T, Charytan D, Strutz F, et al. BMP-7 counteracts TGF-betal-induced epithelial-to-mesenchymal transition and reverses chronic renal injury. Nat Med 2003; 9(7):964-8.

[17.] Archer J. Effect of renoprotective amino acids and a dipeptide on disease progression, nutritional status, and blood-serum phosphate concentration in cats with chronic kidney disease. Research Journal for Veterinary Practitioners 2019; 7(2):39-52.

[18.] Park YJ, Volpe SL, Decker EA. Quantitation of carnosine in humans plasma after dietary consumption of beef. J Agric Food Chem 2005; 53(12):4736-9.

[19.] Everaert I, Taes Y, De Heer E, Baelde H, Zutinic A, Yard B, et al. Low plasma carnosinase activity promotes carnosinemia after carnosine ingestion in humans. Am J Physiol Renal Physiol 2012; 302(12):F1537-44.

[20.] Archer J. Effect of AB070597 on bloodserum creatinine concentration in cats with chronic kidney disease. Research Journal for Veterinary Practitioners 2015; 3(3):58-68. 
[21.] Archer J. Effect of amino acid and peptide complex AB070597 on renal function in dogs with chronic kidney disease. International Animal Health Journal 2018; 5(2):38-42.

[22.] Goldstein RE, Marks SL, Cowgill LD, Kass PH, Rogers QR. Plasma amino acid profiles in cats with naturally acquired chronic renal failure. Am J Vet Res 1999; 60(1):109-13.

[23.] Levey AS, Stevens LA, Schmid CH, Zhang YL, Castro AF 3rd, Feldman HI, et al. A new equation to estimate glomerular filtration rate. Ann Intern Med 2009; 150(9):604-12.

[24.] Tsai CW, Ting IW, Yeh HC, Kuo CC. Longitudinal change in estimated GFR among CKD patients: A 10-year follow-up study of an integrated kidney disease care program in Taiwan. PLoS One 2017; 12(4):e0173843.

[25.] Tukey JW. Exploratory Data Analysis, first ed., Addison -Wesley, Reading, Massachusetts, 1977.

[26.] Nijveldt RJ, Van Leeuwen PA, Van Guldener C, Stehouwer CD, Rauwerda JA, Teerlink T. (2002) Net renal extraction of asymmetrical (ADMA) and symmetrical (SDMA) dimethylarginine in fasting humans. Nephrol Dial Transplant 2002; 17(11):1999-2002.

[27.] Bode-Boger SM, Scalera F, Kielstein JT, Martens-Lobenhoffer J, Breithardt G, Fobker $\mathrm{M}$, et al. Symmetrical dimethylarginine: a new combined parameter for renal function and extent of coronary artery disease. J Am Soc Nephrol 2006; 17(4):1128-34.

[28.] Zoccali C, Bode-Boger S, Mallamaci F, Benedetto F, Tripepi G, Malatino L, et al. Plasma concentration of asymmetrical dimethylarginine and mortality in patients with end-stage renal disease: a prospective study. Lancet 2001; 358(9299):2113-7.
[29.] Klahr S. Can L-arginine manipulation reduce renal disease? Semin Nephrol 1999; 19(3):304-9.

[30.] Thomsen K, Nielsen CB, Flyvbjerg A. Effects of glycine on glomerular filtration rate and segmental tubular handling of sodium in conscious rats. Clin Exp Pharmacol Physiol 2002; 29(5-6):449-54.

[31.] Yatzidis H (2002). A new, superior, single and stable, amino acid and bicarbonate-based glucose-free solution for peritoneal dialysis. Dialysis and Transplantation 2002; 31:143-149.

[32.] Varnier M, Leese GP, Thompson J, Rennie MJ. Stimulatory effect of glutamine on glycogen accumulation in human skeletal muscle. Am J Physiol 1995; 269(2 Pt 1):E309-15.

[33.] Kopple JD, Swendseid ME. Evidence that histidine is an essential amino acid in normal and chronically uremic man. J Clin Invest 1975; 55(5):881-91. 
How to cite this article: Archer J.D. ExploratoryData and Statistical Analyses of AB070597, an Amino Acid/ Peptide Complex, on BloodSerum Creatinine Concentration and Estimated Glomerular Filtration Rate: A Non-Randomized Pilot Trial of Five Humans with Declining Renal Function. Journal of Current Medical Research and Opinion. 2020;749-757. https://doi.org/ 10.15520/jcmro.v3i11.367 